\title{
Colocación inmediata de implantes en zona estética, regeneración ósea guiada en paciente con bulimia nerviosa
}

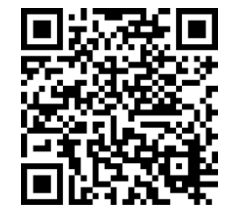

\author{
Jesús Alfredo Aguado Arzola, ${ }^{*}$ Martha Margarita Aguado Arzola, ${ }^{*}$ \\ María de los Ángeles Pietschmann Santamaría, ${ }^{*}$ Valente Alejandro Aguado Herrera ${ }^{\ddagger}$
}

\begin{abstract}
RESUMEN
Este artículo reporta el caso de una paciente de 38 años, la cual ha padecido bulimia nerviosa por más de 20 años teniendo como consecuencia la pérdida de peso de forma importante, por la misma condición podría ser un factor negativo en la osteointegración de una terapia con implantes dentales. Requirió extracción y colocación inmediata de implantes en la zona anterior superior, remoción de granuloma periapical y técnica de regeneración ósea guiada. Los implantes se osteointegración de forma exitosa, se observó radiográficamente una ligera remodelación ósea en dos de los implantes colocados que no interfirió en el resultado final.
\end{abstract}

Palabras clave: Implantes dentales, zona estética, regeneración ósea guiada, bulimia.

\section{INTRODUCCIÓN}

Los trastornos en la conducta alimenticia (TCA) son varios; la anorexia nerviosa y la bulimia nerviosa son trastornos reconocidos entre la población joven. En la población femenina es del $90 \%$ de los casos de entre 12 y 25 años, aunque se tienen casos de personas adultas que sufren estos trastornos alimenticios. Hay diferentes estudios que estiman que de un $0.5 \%$ hasta un $2 \%$ de la población padece este trastorno. ${ }^{1}$

El fenómeno TCA está acentuado en países industrializados. Es una enfermedad de origen psiquiátrico inducida por una fuerte influencia de la moda y la industria del entretenimiento, donde los cuerpos es-

\footnotetext{
* Maestría en Ciencias Odontológicas.

‡ Maestría en Ciencias Odontológicas con acentuación en Periodoncia.
}

Universidad Autónoma de Coahuila Unidad Torreón, Torreón, México.

Recibido: 01 de agosto de 2019. Aceptado: 10 de octubre de 2019.

Este artículo puede ser consultado en versión completa en www.medigraphic.com/periodontologia

\begin{abstract}
This article reports the case of a patient of 38 years who has suffered from bulimia nervosa for more than 20 years resulting in a significant weight loss. This condition could be a negative factor in the osseointegration of a dental implant therapy, which required extraction and immediate implant placement in the upper anterior are, removal of periapical granuloma and guided bone regeneration technique. The implants were osseointegrated successfully. A mild bone remodeling was observed radiographically in two of the implants, which did not interfere in the final result.
\end{abstract}

Keywords: Dental Implants, esthetic zone, guide bone regeneration, bulimia.

tilizados y en extremo delgados inspiran a este grupo de personas psicológicamente vulnerables. ${ }^{2}$

El TCA es una enfermedad de origen psicológico, las personas tienen una distorsión de la imagen corporal y fobia a engordar, se ven a sí mismas con sobrepeso. El paciente sufre una pérdida autoinducida de peso dejando de ingerir alimentos, la cual puede tener consecuencias muy graves. Las afectaciones de este padecimiento van desde las alteraciones psiquiátricas como depresión, ansiedad, trastornos de personalidad; en el área física mantienen una extrema delgadez, muy por debajo de un índice de masa corporal (IMC) $17.5 \mathrm{~kg} / \mathrm{m}^{2}$ (85\% o menos del peso esperado para un sujeto); en casos graves puede provocar desnutrición, amenorrea, osteopenia y en su forma más grave osteoporosis, leucopenia, hipoglucemia, bradicardia, arritmias cardiacas y en algunos casos puede llegar hasta la muerte. ${ }^{1,3}$

Existen dos formas reconocidas de anorexia: una es la restrictiva, en ésta el paciente suprime los alimentos y practica ejercicio; el otro tipo es la no restrictiva (bulimia), en la cual se dan episodios donde el paciente ingiere alimentos e inmediatamente recurre al vómito autoinducido y toma de purgantes 
Rev Mex Periodontol 2019; X (3): 44-48

o medicamentos que puedan ayudar a perder peso. El paciente mantiene oculto este comportamiento. ${ }^{1,4,5}$

La osteopenia o la osteoporosis es una enfermedad en la que hay una baja en la densidad y masa ósea, son condiciones encontradas en pacientes que sufren anorexia nerviosa y éste es un factor a considerar en la terapia para la colocación de implantes dentales y técnicas de regeneración ósea guiada. ${ }^{6-8}$

Existen muy pocas referencias con respecto a la terapia con implantes dentales en pacientes que sufren TCA, pero hay una similitud con pacientes que sufren osteoporosis a quienes se les han colocado implantes. ${ }^{8-11}$

Christopher M. Holahn y colaboradores examinaron a 746 mujeres, a las cuales se les colocaron 3,244 implantes dentales. Tres años después del implante, las puntuaciones de densidad mineral ósea estaban disponibles para 646 implantes (192 pacientes). En este grupo, 57 (29.7\%) fueron diagnosticadas con osteopenia y 41 (21.4\%) con osteoporosis, llegando a la conclusión de que el diagnóstico de osteopenia u osteoporosis no es un factor que aumente el riesgo de fracaso en terapia con implantes. ${ }^{7}$ FCFL de Medeiros y su grupo reportaron en un estudio de metaanálisis que la tasa de supervivencia del implante en pacientes con osteoporosis fue similar a los implantes colocados en pacientes sin osteoporosis. ${ }^{12}$ Amorimand y su equipo colocaron 82 implantes en la mandíbula, 39 de ellos en pacientes con diagnóstico de osteoporosis y 43 en un grupo de control, se perdió sólo un implante que representa el $1.2 \%$, pero no fue posible atribuirle la causa por motivo de la condición de osteoporosis. ${ }^{13}$

\section{DeSCRIPCIÓN DEL CASO CLÍNICO}

Se presentó a la consulta una paciente de 38 años de edad con un historial de trastorno de alimenta-

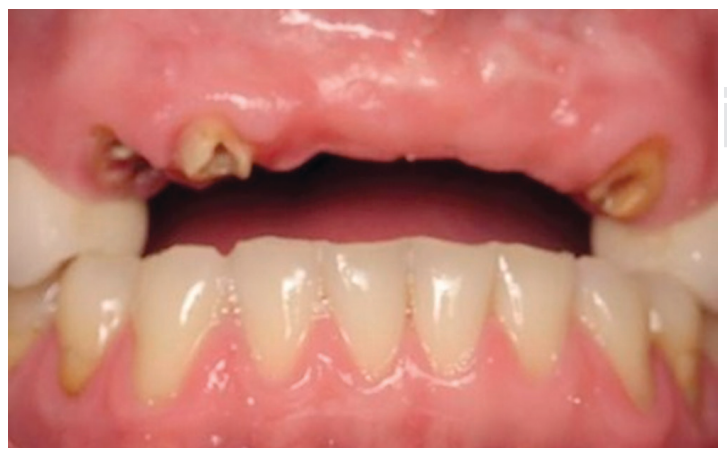

Figura 1: Pilares remanentes.

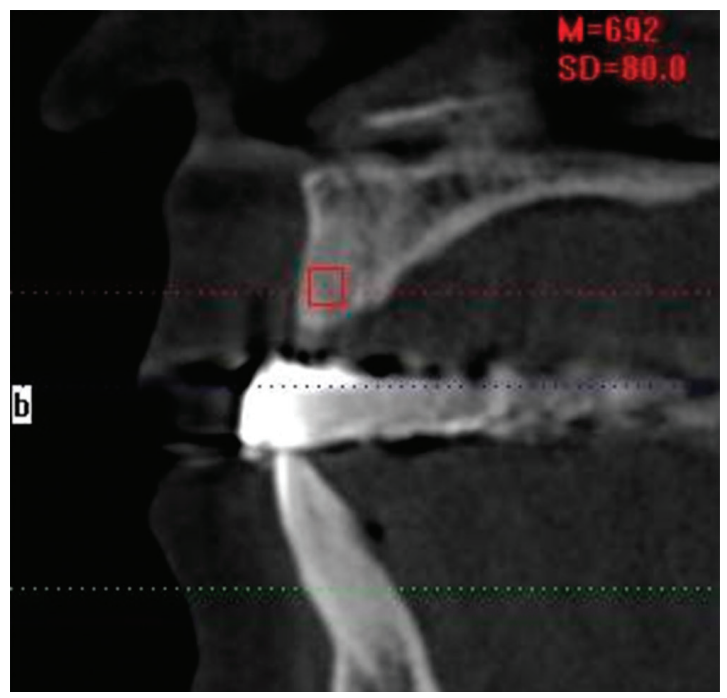

Figura 2: Tomografía, unidades Hounsfield en el área del lateral superior izquierdo.

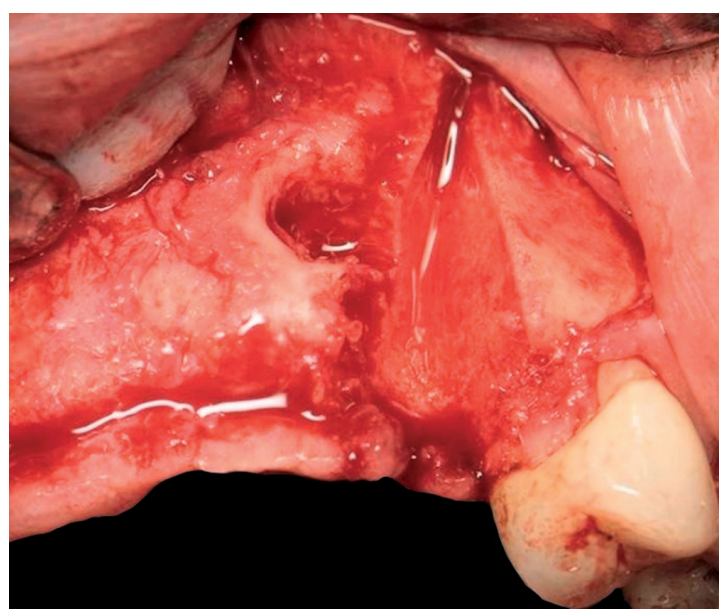

Figura 3: Lesión periapical en el área del lateral y fractura de tabla externa en canino superior izquierdo.

ción (bulimia) con un periodo de más de 22 años de padecerla, teniendo un peso de 42 kilos y una estatura de $1.60 \mathrm{~m}$. La paciente se presenta con un puente cerámico de seis unidades descementado, en el segmento anterior superior los pilares mostraban severa destrucción de su estructura dental por motivo de filtración y caries secundaria (Figura 1). Se realizó una tomografía axial computarizada (TAC) y se detectó una zona radiolúcida en el área apical del incisivo lateral izquierdo. El estudio tomográfico 
Rev Mex Periodontol 2019; X (3): 44-48

mostró mediciones de unidades Hounsfield (HU) teniendo un valor que variaba en la zona de hueso trabecular de 692 a 543 HU (Figura 2).

Debido a los severos desgastes de la estructura dental por efecto químico a causa de la repetitiva conducta de autoprovocación del vómito, el resto de las piezas dentales posteriores superiores y posteriores inferiores presentaban restauraciones cerámicas de cobertura total y obturaciones cervicales en premolares inferiores.

\section{TÉCNICA QUIRÚRGICA}

La cirugía se efectuó después de la administración previa de 1,700 gramos de amoxicilina y $250 \mathrm{mg}$ de ácido clavulánico, se llevó a cabo con sedación intravenosa, se aplicaron anestésicos locales articaína/ epinefrina en una concentración de 1/200,000. Se realizó un colgajo de espesor total con liberatrices verticales distales, respetando las papilas de los

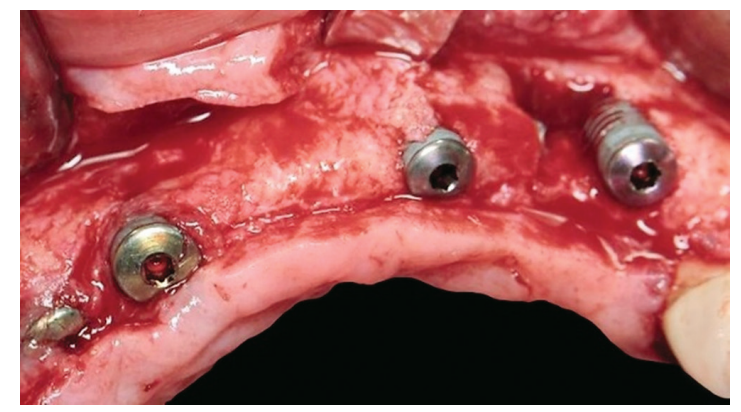

Figura 4: Colocación de implantes dentales Nobel Replace.

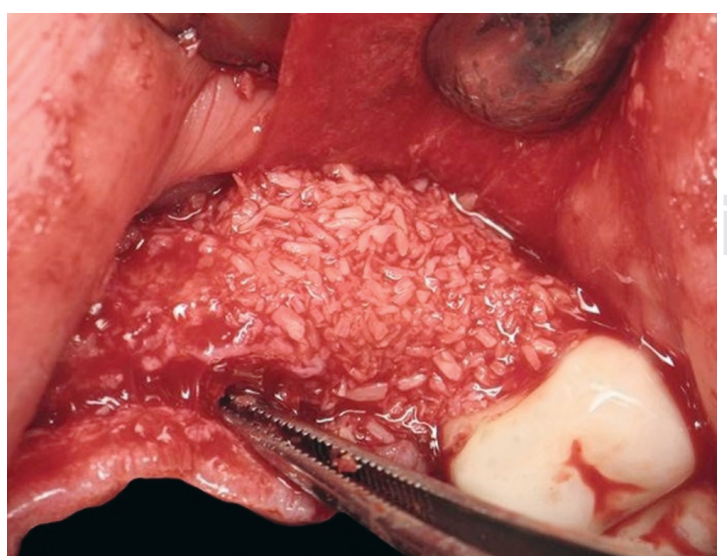

Figura 5: Colocación del aloinjerto.

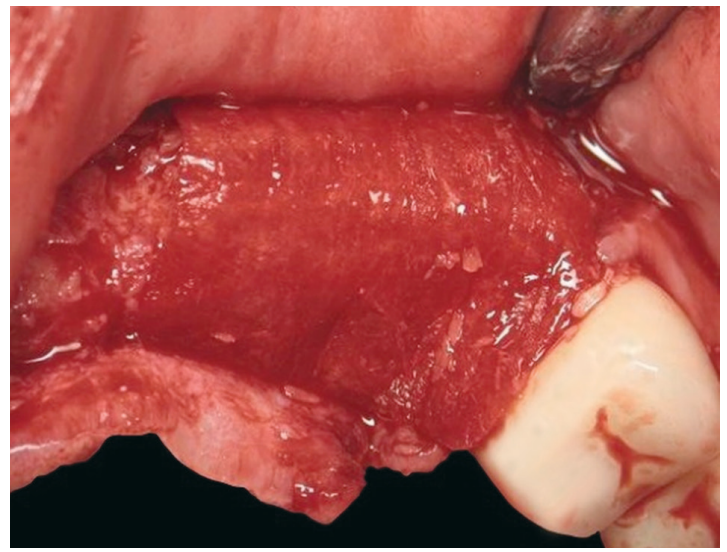

Figura 6: Colocación de membrana de colágeno.

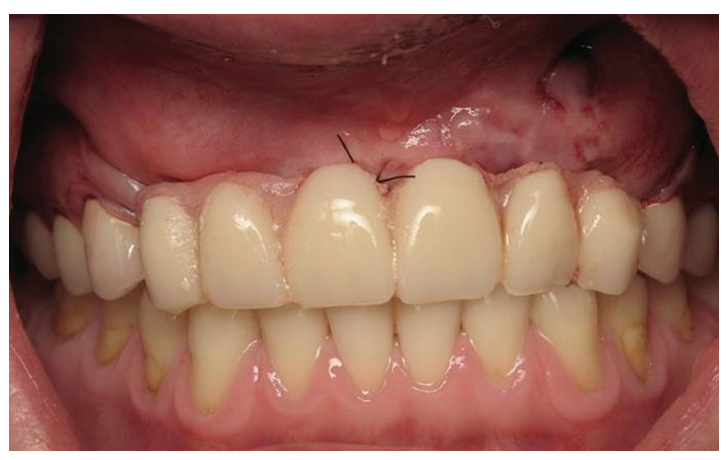

Figura 7: Prótesis parcial removible.

primeros premolares adyacentes. Al colgajo se le hicieron incisiones en el periostio desde la base y en el tercio medio para liberarlo y lograr el cierre del mismo, libre de tensión. Se realizaron las extracciones de los restos radiculares correspondientes a los caninos e incisivo lateral derecho, en el procedimiento se pudo corroborar la presencia de un granuloma periapical en el área del incisivo lateral izquierdo, el cual fue legrado y confirmado como tal con un estudio histopatológico. En el procedimiento de la extracción del canino superior izquierdo se fracturó la tabla ósea externa próxima a la lesión periapical (Figura 3).

Se procedió a la colocación inmediata de cuatro implantes dentales Nobel Replace (Nobel Biocare). En el área del incisivo lateral superior derecho y del área del canino superior izquierdo se colocaron implantes de $4.3 \times 10 \mathrm{~mm}$ y en el área del canino superior derecho y del incisivo central superior se 
colocaron implantes de $3.5 \times 10 \mathrm{~mm}$ (Figura 4). Se colocó aloinjerto óseo en partículas de 500-800 micras (Biograf) (Figura 5), a continuación se cubrió el injerto óseo con una membrana de plasma rica en plaquetas (L-PRP) obtenida de $30 \mathrm{~mL}$ de la sangre recolectada del brazo izquierdo de la paciente, después los injertos fueron cubiertos por membranas de colágeno reabsorbibles de 28 a 36 semanas de permanencia (RCM6), las cuales fueron fijadas con tachuelas de titanio (Figura 6). Se reposicionó el colgajo logrando cierre primario, se suturó con polímero de ácido glicólico 4-0 (Atramat), posterior a la cirugía se continuó con terapia de antibiótico por siete días y la administración de ibuprofeno 400 mg cada ocho horas por seis días. Se optó por colocar una prótesis parcial removible a modo provisional (Figura 7). Las suturas se retiraron a los 15 días después de la intervención.

Se esperó un periodo de cicatrización de seis meses, en el cual no se observaron complicaciones y se procedió a exponer los implantes y colocar tornillos transmucosales. A los 14 días se llevó a cabo la

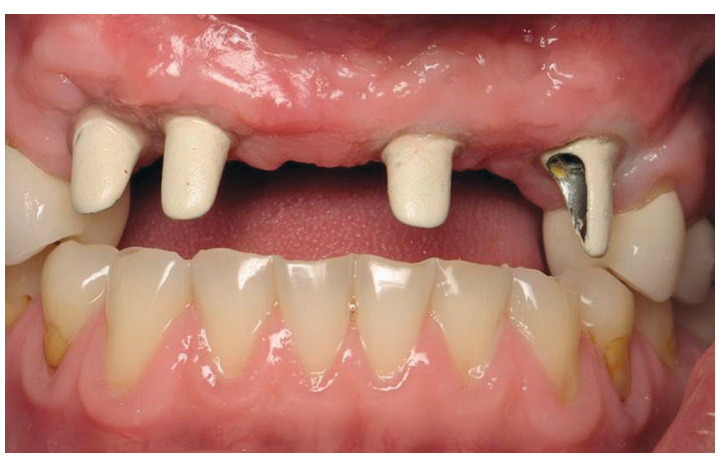

Figura 8: Pilares personalizados.

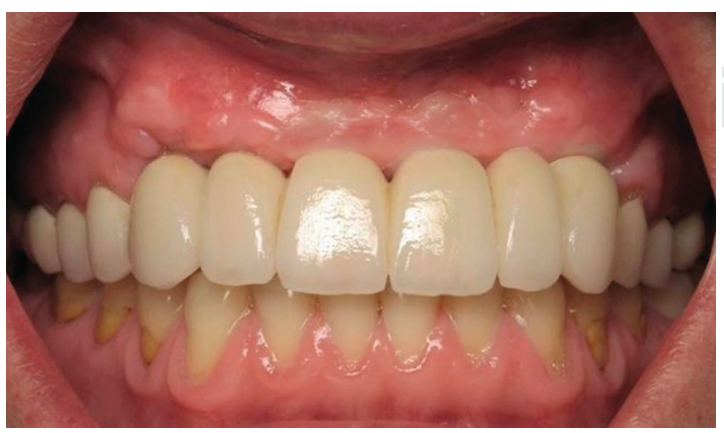

Figura 9: Prótesis fija seis unidades zirconia/cerámica.

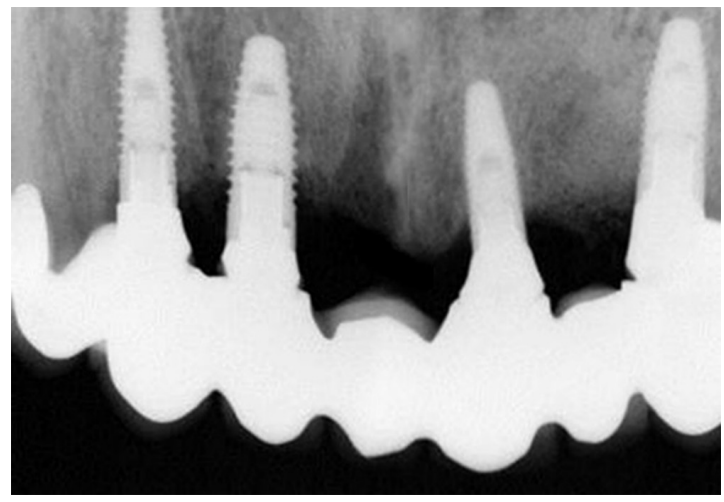

Figura 10: Radiografía de los implantes dentales.

toma de impresión. El plan de tratamiento protésico por el que se optó fue una prótesis fija cementada a base de óxido de zirconio, estratificada con capas cerámicas y pilares personalizados con base metálica y cerámica para optimizar el resultado estético, los cuales fueron torqueados a 35 Newton y la estructura cementada con cemento de resina de curado dual (Figuras 8 y 9).

Se programó a la paciente para mantenimiento. ${ }^{6}$ Fue revisada a los seis, 12 y 24 meses posteriores a la colocación de los implantes ya rehabilitados. Se detectó por medio de una radiografía una ligera remodelación ósea de $1.5 \mathrm{~mm}$ en el implante del incisivo lateral derecho y de $1 \mathrm{~mm}$ del implante correspondiente al incisivo central izquierdo, no existiendo evidencia de periimplantitis ni pérdida de nivel de los tejidos blandos (Figura 10).

\section{Discusión}

Las erosiones dentales, los labios secos o agrietados y el síndrome de ardor en la lengua son manifestaciones comunes en los pacientes con trastornos en la conducta alimenticia, principalmente en pacientes con bulimia nerviosa por la constante provocación del vómito. ${ }^{14}$ Un estudio in vitro por Matsou, E y colaboradores en el año 2011, mostró que la regurgitación de los ácidos del contenido gástrico en la boca de los pacientes bulímicos no influye en las características de las rugosidades de las superficies de los materiales cerámicos de las restauraciones metal cerámicas, lo que sugiere que los implantes dentales se pueden colocar de manera segura en pacientes con bulimia nerviosa. ${ }^{15}$ En un estudio de Ambard 
y Mueninghoff de 2002, se colocaron 14 implantes dentales a una paciente con bulimia nerviosa, éstos se mantuvieron estables en su función después de 12 meses de seguimiento. ${ }^{16}$ Los implantes que son puestos en pacientes con osteoporosis sistémica no presentan contraindicaciones para su colocación, ya que no son un factor de riesgo de osteointegración. ${ }^{17}$

Para mantener a largo plazo los tratamientos con implantes, debemos instaurar un programa de mantenimiento adecuado. Los implantes tienen características específicas que determinan los procedimientos e instrumental a utilizar. En el mantenimiento no sólo motivaremos y formaremos al paciente en técnicas específicas de higiene oral, sino que también debemos vigilar el estado de los tejidos periimplantarios (blandos y duros) y de la prótesis. ${ }^{18}$

\section{Conclusión}

La condición de bulimia nerviosa trae como consecuencia la desnutrición con pérdida de masa muscular y densidad ósea, factores a tomar en cuenta para alcanzar el éxito en una terapia sobre implantes. En el caso clínico aquí descrito, se alcanzó un resultado favorable a pesar de las condiciones de bajo peso de la paciente, sólo con una ligera remodelación ósea inicial que no resultó en una complicación mayor; además, se logró la remodelación ósea en área de la lesión periapical y de la lámina vestibular perdida del área canina.

Se requiere ampliar los estudios de terapia con implantes dentales en pacientes anoréxicos y bulímicos, pero con base en resultados con pacientes que sufren osteoporosis.

No resulta en una contraindicación la terapia de implantes, siempre y cuando existan condiciones mínimas de salud apoyadas en la información de la tomografía para determinar la densidad ósea (HU). Es importante dar a los pacientes instrucciones de higiene y plan de mantenimiento.

Se sugiere hacer investigaciones en pacientes que presenten trastornos alimenticios y dar seguimiento a estos mismos.

\section{Referencias}

1. Fairburn CG, Harrison PJ. Eating disorders. Lancet. 2003; 361: 407-416.

2. Hoek HW, Van Hoeken D. Review of the prevalence and incidence of eating disorders. Int J Eat Disord. 2003; 34 (4): 383-396.
3. Tomba E, Tecuta L, Crocetti E, Squarcio F, Tomei G. Residual eating disorder symptoms and clinical features in remitted and recovered Eating disorder patients: A systematic review with meta-analysis. Int J Eat Disord. 2019; 52 (7): 759-776.

4. Robinson L, Aldridge V, Clark EM, Misra M, Micali N. A systematic review and meta-analysis of the association between eating disorders and bone density. Osteoporos Int. 2016; 27 (6): 1953-1966. doi: 10.1007/s00198-015-3468-4. Epub 2016 Jan 18.

5. Thorton D, Gordon CM. Restrictive eating disorders and skeletal health in adolescent girls and young women. Calcif Tissue Int. 2017; 100 (5): 449-460.

6. Bornstein MM, Cionca N, Mombelli A. Systematic conditions and treatments as risks for implant therapy. Int J Oral Maxillofac Implants. 2009; 24: 12-27.

7. Holahn CM, Koka S, Kennel KA, Weaver AL, Assad DA, Regennitter FJ et al. Effect of osteoporotic status on the survival of titanium dental implants. Int J Oral Maxillofac Implants. 2008; 23: 905-910.

8. Shibli JA, Aguiar KC, Melo L, Ferrari DS, D’Avila S, Iezzi G et al. Histologic analysis of human peri-implant bone in type 1 osteoporosis. J Oral Implantol. 2008; 34 (1): 12-16.

9. Famili P, Zavoral JM. Low skeletal bone mineral density does not affect dental implants. J Oral Implantol. 2015; 41 (5): 550-553.

10. Eder A, Watzek G. Treatment of a patient with severe osteoporosis and chronic polyarthritis with fixed implantsupported prosthesis: a case report. Int J Oral Maxillofacial Implants. 1999; 14: 587-590.

11. Legroux-Gerot I, Vignau J, Collier F, Cortet B. Bone loss associated with anorexia nervosa. Joint Bone Spine. 2005; 72 (6): 489-495.

12. De Medeiros FC, Kudo GA, Leme BG, Saraiva PP, Verri FR, Honório HM et al. Dental implants in patients with osteoporosis: a systematic review meta-analysis. J Int Oral Maxillofac Surg. 2018; 47 (4): 480-491.

13. Amorim MA, Takayama L, Jorgetti V, Pereira RM. Comparative study of axial and femoral bone mineral density and parameters of mandibular bone quality in patients receiving dental implants. Osteoporosis Int. 2007; 18: 703-709.

14. Javed F, Romanos GE. Dental implants in patients with eating disorders. Evidence-based implant dentistry and systematic conditions. John Wiley \& Sons, Inc. 2018.

15. Matsou E, Vouroutzis N, Kontonasaki E, Paraskevopoulos $\mathrm{KM}$, Koidis P. Investigation of the influence of gastric acid on the surface roughness of ceramic materials of metal-ceramic restorations. An in vitro study. Int J Prosthodont. 2011; 24 (1): 26-29.

16. Ambard A, Mueninghoff L. Rehabilitation of bulimic patient using endosteal implants. J Prosthodont. 2002; 11: 176-180.

17. Turhani D, Ohlmeir H, Sutter W, Kielbassa AM. Undesirable course of an oral implant rehabilitation in patient with a long history of bulimia nervosa: case report and review of literature. Quintessence Int. 2019; 50: 68-79.

18. Silverstein LH, Kurtzman GM. Oral hygiene and maintenance of dental implants. Dentistry Today. 2006; 25 (3): 70-75.

Correspondencia:

Martha Margarita Aguado Arzola

Tel: (871) 714-1696

E-mail: marthamaguadoa@gmail.com 